\title{
Non-Commutative Ternary Nambu-Poisson Algebras and Ternary Hom- Nambu-Poisson Algebras
}

\author{
Makhlouf $\mathrm{A}^{1 *}$ and Amri $\mathrm{A}^{2}$ \\ ${ }^{1}$ University of Haute Alsace, Laboratory of Mathematics, IT and Applications, 4 Rue des Freres Lumiere, France \\ ${ }^{2}$ Badji Mokhtar University Annaba, BP 12 Department of Maths, Science Faculty, Algeria
}

\begin{abstract}
The main purpose of this paper is to study non-commutative ternary Nambu-Poisson algebras and their Homtype version. We provide construction results dealing with tensor product and direct sums of two (non- commutative) ternary (Hom-) Nambu-Poisson algebras. Moreover, we explore twisting principle of (non-commutative) ternary Nambu-Poisson algebras along with algebra morphism that lead to construct (non-commutative) ternary HomNambu-Poisson algebras. Furthermore, we provide examples and a 3-dimensional classification of non-commutative ternary Nambu-Poisson algebras.
\end{abstract}

Keywords: Hom-nambu poisson algebra; Ternary nambu poisson; Non-commutative ternary; $n$-ary

\section{Introduction}

In the 70 's, Nambu proposed a generalized Hamiltonian system based on a ternary product, the Nambu-Poisson bracket, which allows to use more than one hamiltonian [1]. More recent motivation for ternary brackets appeared in string theory and M-branes, ternary Lie type structure was closely linked to the super-symmetry and gauge symmetry transformations of the world-volume theory of multiple coincident M2-branes and was applied to the study of Bagger-Lambert theory. Moreover ternary operations appeared in the study of some quarks models. In 1996, quantizations of Nambu-Poisson brackets were investigated [2], it was presented in a novel approach of Zariski, and this quantization is based on the factorization on $\mathbb{R}$ of polynomials of several variables.

The algebraic formulation of Nambu mechanics was discussed [3] and Nambu algebras was studied [4] as a natural generalization of a Lie algebra for higher- order algebraic operations. By definition, Nambu algebra of order $n$ over a field $\mathbb{k}$ of characteristic zero consists of a vector space $V$ over $\mathbb{K}$ together with a $\mathbb{K}$-multilinear skew-symmetric operation $[., \cdots,]:. \Lambda^{n} V \rightarrow V$, called the Nambu bracket that satisfies the following generalization of the Jacobi identity. Namely, for any $x_{1}, \ldots, x_{n-1} \in V$ define an adjoint action $\operatorname{ad}\left(x_{1}, \ldots, x_{n-1}\right): V \rightarrow V$ by $\operatorname{ad}\left(x_{1}, \ldots, x_{\mathrm{n}-1}\right) x_{n}=\left[x_{1}, \ldots, x_{n-1}, x_{n}\right], x_{n} \in V$. Then the fundamental identity is a condition saying that the ad joint action is a derivation with respect the Nambu bracket, i.e. for all $x_{1}, \ldots, x_{n-1}, y_{1}, \ldots, y_{n} \in V$

$$
\operatorname{ad}\left(x_{1}, \ldots, x_{n-1}\right)\left[y_{1}, \ldots, y_{n}\right]=\sum_{k=1}^{n}\left[y_{1}, \ldots, a d\left(x_{1}, \ldots, x_{n-1}\right) y_{k}, \ldots, y_{n}\right] \text {. }
$$

When $n=2$, the fundamental identity becomes the Jacobi identity and we get a definition of a Lie algebra.

Different aspects of Nambu mechanics, including quantization, deformation and various algebraic constructions for Nambu algebras have recently been studied. Moreover a twisted generalization, called Hom-Nambu algebras, was introduced [5]. This kind of algebras called Hom-algebras appeared as deformation of algebras of vector fields using $\sigma$-derivations. The first examples concerned $q$-deformations of Witt and Virasoro algebras. Then Hartwig, Larsson and Silvestrov introduced a general framework and studied Hom-Lie algebras [6], in which Jacobi identity is twisted by a homomorphism. The corresponding associative algebras, called Hom-associative algebras were introduced [7]. Non-commutative Hom-Poisson algebras were discussed [8]. Likewise, $n$-ary algebras of Hom-type were introduced [5,9-13].

We aim in this paper to explore and study non-commutative ternary Nambu-Poisson algebras and their Hom-type version. The paper includes five Sections. In the first one, we summarize basic definitions of (non-commutative) ternary Nambu- Poisson algebras and discuss examples. In the second Section, we recall some basics about Homalgebra structures and introduce the notion of (non-commutative) ternary Hom-Nambu-Poisson algebra. Section 3 is dedicated to construction of (non-commutative) ternary Hom-Nambu-Poisson algebras using direct sums and tensor products. In Section 4, we extend twisting principle to ternary Hom-Nambu- Poisson algebras. It is used to build new structures with a given ternary (Hom-) Nambu-Poisson algebra and algebra morphism. This process is used to construct ternary Hom-Nambu-Poisson algebras corresponding to the ternary algebra of polynomials where the bracket is defined by the Jacobian. We provide in the last section a classification of 3-dimensional ternary NambuPoisson algebras and then compute corresponding Hom-NambuPoisson algebras using twisting principle. Notice that a complete classification of 3-dimensional Hom-Nambu-Poisson algebras is difficult to obtain since so far the classification of 3-dimensional HomNambu-Lie algebras is not known.

\section{Ternary (Non-Commutative) Nambu-Poisson Algebra}

In the section we review some basic definitions and fix notations. In the sequel, $A$ denotes a vector space over $\mathbb{K}$, where $\mathbb{K}$ is an algebraically closed field of characteristic zero. Let $\mu: A \times A \rightarrow A$ be a bilinear map, we denote by $\mu^{o p}: A^{\times 2} \rightarrow A$ the opposite map, i.e., $\mu^{o p}=\mu$ o $\tau$ where $\tau: A^{\times 2} \rightarrow A^{\times 2}$ interchanges the two variables.

*Corresponding author: Makhlouf A, University of Haute Alsace, Laboratory of Mathematics, IT and Applications, 4 Rue des Freres Lumiere, 68093 Mulhouse Cedex, France, Tel: +33 3893365 00; E-mail: abdenacer.makhlouf@uha.fr

Received October 17, 2014; Accepted June 30, 2015; Published July 07, 2015

Citation: Makhlouf A, Amri A (2015) Non-Commutative Ternary Nambu-Poisson Algebras and Ternary Hom-Nambu-Poisson Algebras. J Generalized Lie Theory Appl 9: 221. doi:10.4172/1736-4337.1000221

Copyright: (C) 2015 Makhlouf A, et al. This is an open-access article distributed under the terms of the Creative Commons Attribution License, which permits unrestricted use, distribution, and reproduction in any medium, provided the original author and source are credited. 
A ternary algebra is given by a pair $(A, m)$, where $m$ is a ternary operation on $A$, that is a trilinear map $m: A \times A \times A \rightarrow A$, which is denoted sometimes by brackets.

Definition 1.1. A ternary Nambu algebra is a ternary algebra $(A,\{,\}$, satisfying the fundamental identity defined as

$$
\begin{aligned}
& \left\{x_{1}, x_{2},\left\{x_{3}, x_{4}, x_{5}\right\}\right\}= \\
& \left\{\left\{x_{1}, x_{2}, x_{3}\right\}, x_{4}, x_{5}\right\}+\left\{x_{3},\left\{x_{1}, x_{2}, x_{4}\right\}, x_{5}\right\}+\left\{x_{3}, x_{4},\left\{x_{1}, x_{2}, x_{5}\right\}\right\}
\end{aligned}
$$

for all $x_{1}, x_{2}, x_{3}, x_{4}, x_{5} \in A$.

This identity is sometimes called Filippov identity or Nambu identity, and it is equivalent to the identity (0.1) with $n=3$.

A ternary Nambu-Lie algebra or 3-Lie algebra is a ternary Nambu algebra for which the bracket is skew-symmetric, that is for all $\sigma \in S_{3}$, where $S_{3}$ is the permutation group,

$$
\left[x_{\sigma(1)}, x_{\sigma(2)}, x_{\sigma(3)}\right]=\operatorname{Sgn}(\sigma)\left[x_{1}, x_{2}, x_{3}\right] \text {. }
$$

Let $A$ and $A^{\prime}$ be two ternary Nambu algebras (resp. Nambu-Lie algebras). A linear map $f: A \rightarrow A^{\prime}$ is a morphism of a ternary Nambu algebras (resp. ternary Nambu-Lie algebras) if it satisfies $f\left(\{x, y, z\}_{A}\right)=\{f$ $(x), f(y), f(z)\} A^{\prime}$.

Example 1.2. The polynomials of variables $x_{1}, x_{2}, x_{3}$ with the ternary operation defined by the Jacobian function:

$$
\left\{f_{1}, f_{2}, f_{3}\right\}=\left|\begin{array}{lll}
\frac{\partial f_{1}}{\partial x_{1}} & \frac{\partial f_{1}}{\partial x_{2}} & \frac{\partial f_{1}}{\partial x_{3}} \\
\frac{\partial f_{2}}{\partial x_{1}} & \frac{\partial f_{2}}{\partial x_{2}} & \frac{\partial f_{2}}{\partial x_{3}} \\
\frac{\partial f_{3}}{\partial x_{1}} & \frac{\partial f_{3}}{\partial x_{2}} & \frac{\partial f_{3}}{\partial x_{3}}
\end{array}\right|,
$$

is a ternary Nambu-Lie algebra.

Example 1.3. Let $V=\mathbb{R}^{4}$ be the 4-dimensional oriented Euclidian space over $\mathbb{R}$. The bracket of 3 vectors $\vec{x}, \vec{y}, \vec{z}$ is given by

$$
[x, y, z]=\vec{x} \times \vec{y} \times \vec{z}=\left|\begin{array}{cccc}
x_{1} & y_{1} & z_{1} & e_{1} \\
x_{2} & y_{2} & z_{2} & e_{2} \\
x_{3} & y_{3} & z_{3} & e_{3} \\
x_{4} & y_{4} & z_{4} & e_{4}
\end{array}\right|,
$$

Where $\left\{e_{1}, e_{2}, e_{3}, e_{4}\right\}$ is a basis of $\mathbb{R}^{4}$ and $\vec{x}=\sum_{i=1}^{3} x_{i} \overrightarrow{e_{i}}, \vec{y}=\sum_{i=1}^{3} y_{i} \vec{e}_{i}$ and $\vec{z}=\sum_{i=1}^{3} z_{i} \vec{e}_{i}$. Then $(V,[., .,]$.$) is a ternary Nambu - lie algebra.$

Now, we introduce the notion of (non-commutative) ternary Nambu-Poisson algebra.

Definition 1.4. A non-commutative ternary Nambu-Poisson algebra is a triple $(A, \mu,\{., .,\}$.$) consisting of a \mathbb{K}$-vector space $A$, a bilinear map $\mu: A \times A \rightarrow A$ and a trilinear map $\{., .,\}:. A \otimes A \otimes A \rightarrow$ A such that

(1) $(A, \mu)$ is a binary associative algebra,

(2) $(A,\{., .,\}$.$) is a ternary Nambu-Lie algebra,$

(3) the following Leibniz rule

$\left\{x_{1}, x_{2}, \mu\left(x_{3}, x_{4}\right)\right\}=\mu\left(x_{3},\left\{x_{1}, x_{2}, x_{4}\right\}\right)+\mu\left(\left\{x_{1}, x_{2}, x_{3}\right\}, x_{4}\right)$

holds for all $x_{1}, x_{2}, x_{3} \in A$.

A ternary Nambu-Poisson algebra is a non-commutative ternary
Nambu-Poisson algebra $(A, \mu,\{., .,\}$.$) for which \mu$ is commutative, then $\mu$ is commutative unless otherwise stated.

In a (non-commutative) ternary Nambu-Poisson algebra, the ternary bracket $\{., .,$.$\} is called Nambu-Poisson bracket.$

Similarly, a non-commutative $n$-ary Nambu-Poisson algebra is a triple $(A, \mu,\{., \cdots,\}$.

where $(A,\{., \cdots,\}$.$) defines an n$-Lie algebra satisfying similar Leibniz rule with respect to $\mu$.

A morphism of (non-commutative) ternary Nambu-Poisson algebras is a linear map that is a morphism of the underlying ternary Nambu-Lie algebras and associative algebras.

Example 1.5. Let $C^{\infty}\left(\mathbb{R}^{3}\right)$ be the algebra of $C^{\infty}$ functions on $\mathbb{R}^{3}$ and $x_{1}, x_{2}, x_{3}$ the coordinates on $\mathbb{R}^{2}$. We define the ternary brackets as in (1.2), then $\left(C^{\infty}\left(\mathbb{R}^{3}\right),\{., .,\}.\right)$ is a ternary Nambu-Lie algebra. In addition the bracket satisfies the Leibniz rule: $f g,\left\{f g, f_{2}, f_{3}\right\}=f\left\{g, f_{2}, f_{3}\right\}+\left\{f, f_{2}, f_{3}\right\} g$ where $f, g, f_{2}, f_{3} \in C^{\infty}\left(\mathbb{R}^{3}\right)$ and the multiplication being the point wise multiplication that is $f g(x)=f(x) g(x)$. Therefore, the algebra is a ternary Nambu-Poisson algebra.

This algebra was considered already in 1973 by Nambu [9] as a possibility of extending the Poisson bracket of standard hamiltonian mechanics to bracket of three functions defined by the Jacobian. Clearly, the Nambu bracket may be generalized further to a Nambu-Poisson allowing for an arbitrary number of entries.

In particular, the algebra of polynomials of variables $x_{1}, x_{2}, x_{3}$ with the ternary operation defined by the Jacobian function in (1.2), is a ternary Nambu-Poisson algebra.

Remark 1.6. The $n$-dimensional ternary Nambu-Lie algebra of Example 1.3 does not carry a non-commutative Nambu-Poison algebra structure except that one given by a trivial multiplication.

\section{Hom-type (Non-Commutative) Ternary Nambu- Poisson Algebras}

In this section, we present various Hom-algebra structures. The main feature of Hom-algebra structures is that usual identities are deformed by an endomorphism and when the structure map is the identity, we recover the usual algebra structure.

A Hom-algebra (resp. ternary Hom-algebra) is a triple $(A, v, \alpha)$ consisting of a $\mathbb{K}$-vector space $A$, a bilinear map $v: A \times A \rightarrow A$ (resp. a trilinear map $v: A \times A \times A \rightarrow A$ ) and a linear map $\alpha: \mathrm{A} \rightarrow A$. A Homalgebra $(A, \mu, \alpha)$ is said to be multiplicative if $\alpha$ o $\mu=\mu \mathrm{o} \alpha^{\otimes 2}$ and it is called commutative if $\mu=\mu^{o p}$. A ternary Hom-algebra $(A, m, \alpha)$ is said to be multiplicative if $\alpha$ o $m=m o \alpha^{\otimes 3}$. Classical algebras (resp. ternary algebras) are regarded as Hom-algebras (resp. ternary Hom-algebras) with identity twisting map. We will often use the abbreviation $x y$ for $\mu(x, y)$ when there is no ambiguity. For a linear map $\alpha: A \rightarrow A$, denote by $\alpha^{n}$ the $n$-fold composition of $n$-copies of $\alpha$, with $\alpha^{0} \equiv I d$.

Definition 2.1. A Hom-algebra $(A, \mu, \alpha)$ is a Hom-associative algebra if it satisfies the Hom-associativity condition, that is

$\mu(\alpha(x), \mu(y, z))=\mu(\mu(x, y), \alpha(z))$ for all $x, y, z \in A$.

Remark 2.2. When $\alpha$ is the identity map, we recover the classical associativity condition, then usual associative algebras.

Definition 2.3. A ternary Hom-Nambu algebra is a triple $(A,\{., .,\},. \tilde{\alpha})$ consisting of a $\mathbb{K}$-vector space $A$, a ternary map $\{.$, ., 
.\}: A \times A \times A \rightarrow A$ and a pair of $\tilde{\alpha}=\left(\alpha_{1}, \alpha_{2}\right)$ where $\alpha_{1}, \alpha_{2}: A \rightarrow A$, satisfying [5]

$$
\begin{aligned}
& \left\{\alpha_{1}\left(x_{1}\right), \alpha_{2}\left(x_{2}\right),\left\{x_{3}, x_{4}, x_{5}\right\}\right\}=\left\{\left\{x_{1}, x_{2}, x_{3}\right\}, \alpha_{1}\left(x_{4}\right), \alpha_{2}\left(x_{5}\right)\right\}+ \\
& \left\{\alpha_{1}\left(x_{3}\right),\left\{x_{1}, x_{2}, x_{4}\right\}, \alpha_{2}\left(x_{5}\right)\right\}+\left\{\alpha_{1}\left(x_{3}\right), \alpha_{2}\left(x_{4}\right),\left\{x_{1}, x_{2}, x_{5}\right\}\right\} .
\end{aligned}
$$

We call the above condition the ternary Hom-Nambu identity.

Generally, the $n$-ary Hom-Nambu algebras are defined by an n-ary bracket and maps $\alpha_{1}, \cdots, \alpha_{\mathrm{n}-1}$, satisfying the following Hom-Nambu identity

$$
\begin{aligned}
& \left\{\alpha_{1}\left(x_{1}\right), \ldots, \alpha_{n-1}\left(x_{n-1}\right),\left\{x_{n}, \ldots, x_{2 n-1}\right\}\right\} \\
& =\sum_{i=n}^{2 n-1}=\left\{\alpha_{1}\left(x_{n}\right), \ldots, \alpha_{i-n}\left(x_{i-1}\right),\left\{x_{1}, \ldots, x_{n-1}, x_{i}\right\}, \alpha_{i-n+1}\left(x_{i+1}\right) \ldots, \alpha_{n-1}\left(x_{2 n-1}\right)\right\}
\end{aligned}
$$

for all $\left(x_{1}, \ldots, x_{2 n-1}\right) \in A^{2 n-1}$.

Remark 2.4. A Hom-Nambu algebra is a Hom-Nambu-Lie algebra if the bracket is skew-symmetric.

We introduce now the definition of non-commutative ternary Hom-Nambu-Poisson algebra in its general form, involving three linear maps. Next, we will discuss the class in which these three maps are equal. This particular case suits to provide a twisting construction.

Definition 2.5. A non-commutative ternary Hom-Nambu-Poisson algebra is a tuple $(A, \mu, \beta,\{., .,\},. \tilde{\alpha})$ consisting of a vector space $A$, a ternary operation $\{., .,\}:. A \times A \times A \rightarrow A$, a binary operation $\mu: A \times A \rightarrow A$, a pair of linear maps $\tilde{\alpha}=\left(\alpha_{1}, \alpha_{2}\right)$

where $\alpha_{1}, \alpha_{2}: A \rightarrow A$, and a linear map $\beta: A \rightarrow A$ such that:

$(A, \mu, \beta)$ is a binary Hom-associative algebra,

$(A,\{., .,\},. \tilde{\alpha})$ is a ternary Hom-Nambu-Lie algebra,

$\left\{\mu\left(x_{1}, x_{2}\right), \alpha_{1}\left(x_{3}\right), \alpha_{2}\left(x_{4}\right)\right\}=\mu\left(\beta\left(x_{1}\right),\left\{x_{2}, x_{3}, x_{4}\right\}\right)+\mu\left(\left\{x_{1}, x_{3}, x_{4}\right\}, \beta\left(x_{2}\right)\right)$.

The third condition is called Hom-Leibniz identity.

When, all the linear maps are equal $\alpha=\alpha_{1}=\alpha_{2}=\beta$, we refer to the ternary

Hom-Nambu-Poisson algebra by a quadruple $(A, \mu,\{., .,\},. \alpha)$.

Remark 2.6. Notice that $\mu$ is not assumed to be commutative. When $\mu$ is a commutative multiplication, then $(A, \mu, \beta,\{., .,\},. \tilde{\alpha})$ is said to be a ternary Hom-Nambu-Poisson algebra.

We recover the classical (non-commutative) ternary NambuPoisson algebra when $\alpha_{1}=\alpha_{2}=\beta=I d$.

Similarly, a non-commutative n-ary Hom-Nambu-Poisson algebra is a tuple $(A, \mu, \beta,\{., \ldots,\},. \tilde{\alpha})$ where $(A,\{., \ldots,\},. \tilde{\alpha})$ with linear maps $\tilde{\alpha}=\left(\alpha_{1}, \ldots, \alpha_{n-1}\right)$ that defines an $n$-ary Hom-Nambu-Lie algebra satisfying similar Leibniz rule with respect to $(A, \mu, \beta)$.

In the sequel we will mainly interested in the class of noncommutative ternary Nambu-Poisson algebras where $\alpha=\alpha_{1}=\alpha=\beta$.

Definition 2.7. Let $(A, \mu,\{., .,\},. \alpha)$ be a (non-commutative) ternary Hom-Nambu-Poisson algebra. It is said to be multiplicative if

$\alpha(\{x 1, x 2, x 3\})=\left\{\alpha\left(x_{1}\right), \alpha\left(x_{2}\right), \alpha\left(x_{3}\right)\right\}$,

$\alpha \mathrm{o} \mu=\mu \mathrm{o} \alpha \otimes 2$.

If in addition $\alpha$ is bijective then it is called regular.

Definition 2.8. Let $(A, \mu,\{., .,\},. \alpha)$ and $\left(A^{\prime}, \mu^{\prime},\{., .,\}^{\prime}, \alpha^{\prime}\right)$ be two (non-commutative) ternary Hom-Nambu-Poisson algebras. A linear map $f: A \rightarrow A^{\prime}$ is a morphism of (non-commutative) ternary HomNambu-Poisson algebras if it satisfies for all $x_{1}, x_{2}, x_{3} \in A$ :

$$
\begin{aligned}
& f\left(\left\{x_{1}, x_{2}, x_{3}\right\}\right)=\left\{f\left(x_{1}\right), f\left(x_{2}\right), f\left(x_{3}\right)\right\}^{\prime}, \\
& f \circ \mu=\mu^{\prime} \text { o } f^{\otimes 2} \\
& f \circ \alpha=\alpha^{\prime} \text { o } f .
\end{aligned}
$$

It said to be a weak morphism if hold only the two first conditions.

\section{Tensor Product and Direct Sums}

In this section we discuss direct sums and define tensor product of ternary (non-commutative) Hom-Nambu-Poisson algebra and a totally Hom-associative sym-metric ternary algebra. In the following, we define a direct sum of two ternary (non-commutative) HomNambu-Poisson algebras.

Theorem 3.1. Let $\left(A_{1}, \mu_{1},\{., .,\}_{1}, \alpha_{1}\right)$ and $\left(A_{2}, \mu_{2},\{., .,\}_{2}, \alpha_{2}\right)$ be two ternary (non-commutative) Hom-Nambu-Poisson algebras. Let $\mu_{A 1 \oplus A 2}$ be a bilinear map on $A_{1} \oplus A_{2}$ defined for $x_{1}, y_{1}, z_{1} \in A_{1}$ and $x_{2}, y_{2}, z_{2} \in$ $A_{1}$ by $\mu\left(x_{1}+x_{2}, y_{1}+y_{2}\right)=\mu_{1}\left(x_{1}, y_{1}\right)+\mu_{2}\left(x_{2}, y_{2}\right),\{., .,.\} A_{1} \oplus A_{2}$ a trilinear map defined by $\left\{x_{1}+x_{2}, y_{1}+y_{2}, z_{1}+z_{2}\right\}_{A_{1} \oplus A_{2}}=\left\{x_{1}, y_{1}, z_{1}\right\}_{1}+\left\{x_{2}, y_{2}, z_{2}\right\}_{2}$ and $\alpha_{A_{1} \oplus A_{2}}$ a linear map defined by $\alpha_{A_{1} \oplus A_{2}}\left(x_{1}+x_{2}\right)=\alpha_{1}\left(x_{1}\right)+\alpha_{2}\left(x_{2}\right)$.

Then $\left(A_{1} \oplus A_{2}, \mu_{A_{1} \oplus A_{2}},\{., ., .\}_{A_{1} \oplus A_{2}}, \alpha_{A_{1} \oplus A_{2}}\right)$ is a ternary (noncommutative) Hom-Nambu-Poisson algebra.

Proof. The commutativity of $\mu_{A_{1} \oplus A_{2}}$ is obvious since $\mu_{1}$ and $\mu_{2}$ are commutative. The skew-symmetry of the bracket follows from the skew-symmetry of $\{., ., .\}_{1}$ and $\{., ., .\}_{2}$. So it remains to check the Homassociativity, the Hom-Nambu and the Hom-Leibniz identities. For Hom-associativity identity, we have

$$
\begin{aligned}
& \mu_{A_{1} \oplus A_{2}}\left(\mu_{A_{1} \oplus A_{2}}\left(x_{1}+x_{1}^{\prime}, x_{2}+x_{2}^{\prime}\right), \alpha_{A_{1} \oplus A_{2}}\left(x_{3}+x_{3}^{\prime}\right)\right) \\
& =\mu_{A_{1} \oplus A_{2}}\left(\mu_{1}\left(x_{1}, x_{2}\right)+\mu_{2}\left(x_{1}^{\prime}, x_{2}^{\prime}\right), \alpha_{1}\left(x_{3}\right)+\alpha_{2}\left(x_{3}^{\prime}\right)\right) \\
& =\mu_{1}\left(\mu_{1}\left(x_{1}, x_{2}\right), \alpha_{1}\left(x_{3}\right)\right)+\mu_{2}\left(\mu_{2}\left(x_{1}^{\prime}, x_{2}^{\prime}\right), \alpha_{2}\left(x_{3}^{\prime}\right)\right) \\
& =\mu_{1}\left(\alpha_{1}\left(x_{1}\right), \mu_{1}\left(x_{2}, x_{3}\right)\right)+\mu_{2}\left(\alpha_{2}\left(x_{1}^{\prime}\right), \mu_{2}\left(x_{2}^{\prime}, x_{3}^{\prime}\right)\right) \\
& =\mu_{A_{1} \oplus A_{2}}\left(\alpha_{1}\left(x_{1}\right)+\alpha_{2}\left(x_{1}^{\prime}\right), \mu_{1}\left(x_{2}, x_{3}\right)+\mu_{2}\left(x_{2}^{\prime}, x_{3}^{\prime}\right)\right) \\
& =\mu_{A_{1} \oplus A_{2}}\left(\alpha_{A_{1} \oplus A_{2}}\left(x_{1}, x_{1}^{\prime}\right), \mu_{A_{1} \oplus A_{2}}\left(x_{2}+x_{2}^{\prime}, x_{3}+x_{3}^{\prime}\right)\right)
\end{aligned}
$$

Now we prove the Hom-Nambu identity

$$
\begin{aligned}
& \left\{\alpha_{A_{1} \oplus A_{2}}\left(x_{1}+x_{1}^{\prime}\right), \alpha_{A_{1} \oplus A_{2}}\left(x_{2}+x_{2}^{\prime}\right),\left\{x_{3}+x_{3}^{\prime}, x_{4}+x_{4}^{\prime}, x_{5}+x_{5}^{\prime}\right\}_{A_{1} \oplus A_{2}}\right\}_{A_{1} \oplus A_{2}} \\
& =\left\{\alpha_{1}\left(x_{1}\right)+\alpha_{2}\left(x_{1}^{\prime}\right), \alpha_{1}\left(x_{2}\right)+\alpha_{2}\left(x_{2}^{\prime}\right),\left\{x_{3}, x_{4}, x_{5}\right\}_{1}+\left\{x_{3}^{\prime}, x_{4}^{\prime}, x_{5}^{\prime}\right\}_{2}\right\}_{A_{1} \oplus A_{2}} \\
& =\left\{\alpha_{1}\left(x_{1}\right), \alpha_{1}\left(x_{2}\right),\left\{x_{3}, x_{4}, x_{5}\right\}_{1}\right\}_{1}+\left\{\alpha_{2}\left(x_{1}^{\prime}\right), \alpha_{2}\left(x_{2}^{\prime}\right),\left\{x_{3}^{\prime}, x_{4}^{\prime}, x_{5}^{\prime}\right\}_{2}\right\}_{2} \\
& =\left\{\left\{x_{1}, x_{2}, x_{3}\right\}_{1}, \alpha_{1}\left(x_{4}\right), \alpha_{1}\left(x_{5}\right)\right\}_{1}+\left\{\alpha_{1}\left(x_{3}\right),\left\{x_{1}, x_{2}, x_{4}\right\}_{1}, \alpha_{1}\left(x_{5}\right)\right\}_{1} \\
& +\left\{\alpha_{1}\left(x_{3}\right), \alpha_{1}\left(x_{4}\right),\left\{x_{1}, x_{2}, x_{5}\right\}_{1}\right\}_{1}+\left\{\left\{x_{1}^{\prime}, x_{2}^{\prime}, x_{3}^{\prime}\right\}_{2}, \alpha_{2}\left(x_{4}^{\prime}\right), \alpha_{2}\left(x_{5}^{\prime}\right)\right\}_{2} \\
& +\left\{\alpha_{2}\left(x_{3}^{\prime}\right),\left\{x_{1}^{\prime}, x_{2}^{\prime}, x_{4}^{\prime}\right\}_{2}, \alpha_{2}\left(x_{5}^{\prime}\right)\right\}_{2}+\left\{\alpha_{2}\left(x_{3}^{\prime}\right), \alpha_{2}\left(x_{4}^{\prime}\right),\left\{x_{1}^{\prime}, x_{2}^{\prime}, x_{5}^{\prime}\right\}_{2}\right\}_{2} \\
& =\left\{\left\{x_{1}, x_{2}, x_{3}\right\}_{1}+\left\{x_{1}^{\prime}, x_{2}^{\prime}, x_{3}^{\prime}\right\}_{2}, \alpha_{1}\left(x_{4}\right)+\alpha_{2}\left(x_{4}^{\prime}\right), \alpha_{1}\left(x_{5}\right)+\alpha_{2}\left(x_{5}^{\prime}\right)\right\}_{A_{1} \oplus A_{2}} \\
& +\left\{\alpha_{1}\left(x_{3}\right)+\alpha_{2}\left(x_{3}^{\prime}\right),\left\{x_{1}, x_{2}, x_{4}\right\}_{1}+\left\{x_{1}^{\prime}, x_{2}^{\prime}, x_{4}^{\prime}\right\}_{2}, \alpha_{1}\left(x_{5}\right)+\alpha_{2}\left(x_{5}^{\prime}\right)\right\}_{A_{1} \oplus A_{2}} \\
& +\left\{\alpha_{1}\left(x_{3}\right)+\alpha_{2}\left(x_{3}^{\prime}\right), \alpha_{1}\left(x_{3}\right)+\alpha_{2}\left(x_{3}^{\prime}\right),\left\{x_{1}, x_{2}, x_{5}\right\}_{1}+\left\{x_{1}^{\prime}, x_{2}^{\prime}, x_{5}^{\prime}\right\}_{2}\right\}_{A_{1} \oplus A_{2}} \\
& =\left\{\left\{x_{1}+x_{1}^{\prime}, x_{2}+x_{2}^{\prime}, x_{3}+x_{3}^{\prime}\right\}_{A_{1} \oplus A_{2}}, \alpha_{A_{1} \oplus A_{2}}\left(x_{4}+x_{4}^{\prime}\right), \alpha_{A_{1} \oplus A_{2}}\left(x_{5}+x_{5}^{\prime}\right)\right\}_{A 1 \oplus A_{2}} \\
& +\left\{\alpha_{A_{1} \oplus A_{2}}\left(x_{3}+x_{3}^{\prime}\right),\left\{x_{1}+x_{1}^{\prime}, x_{2}+x_{2}^{\prime}, x_{4}+x_{4}^{\prime}\right\}_{A_{1} \oplus A_{2}}, \alpha_{A_{1} \oplus A_{2}}\left(x_{5}+x_{5}^{\prime}\right)\right\}_{A \oplus A_{2}} \\
& +\left\{\alpha_{A_{1} \oplus A_{2}}\left(x_{3}+x_{3}^{\prime}\right), \alpha_{A_{1} \oplus A_{2}}\left(x_{4}+x_{4}^{\prime}\right),\left\{x_{1}+x_{1}^{\prime}, x_{2}+x_{2}^{\prime}, x_{5}+x_{5}^{\prime}\right\}_{A_{1} \oplus A_{2}}\right\}_{A_{1} \oplus A_{2}}
\end{aligned}
$$


Finally, for Hom-Leibniz identity we have

$$
\begin{aligned}
& \left\{\mu_{A_{1} \oplus A_{2}}\left(x_{1}+x_{1}^{\prime}, x_{2}+x_{2}^{\prime}\right), \alpha_{A_{1} \oplus A_{2}}\left(x_{3}, x_{3}^{\prime}\right), \alpha_{A_{1}+A_{2}}\left(x_{4}, x_{4}^{\prime}\right)\right\}_{A_{1} \oplus A_{2}} \\
& =\left\{\mu_{1}\left(x_{1}, x_{2}\right)+\mu_{2}\left(x_{1}^{\prime}, x_{2}^{\prime}\right), \alpha_{1}\left(x_{3}\right)+\alpha_{2}\left(x_{3}^{\prime}\right), \alpha_{1}\left(x_{4}\right)+\alpha_{2}\left(x_{4}^{\prime}\right)\right\}_{A_{1} \oplus A_{2}} \\
& =\left\{\mu_{1}\left(x_{1}, x_{2}\right), \alpha_{1}\left(x_{3}\right), \alpha_{1}\left(x_{4}\right)\right\}_{1}+\left\{\mu_{2}\left(x_{1}^{\prime}, x_{2}^{\prime}\right), \alpha_{2}\left(x_{3}^{\prime}\right), \alpha_{2}\left(x_{4}^{\prime}\right)\right\}_{2} \\
& =\mu_{1}\left(\alpha_{1}\left(x_{1}\right),\left\{x_{2}, x_{3}, x_{4}\right\}_{1}\right)+\mu_{1}\left(\left\{x_{1}, x_{3}, x_{4}\right\}_{1}, \alpha_{1}\left(x_{2}\right)\right) \\
& +\mu_{2}\left(\alpha_{2}\left(x_{1}^{\prime}\right),\left\{x_{2}^{\prime}, x_{3}^{\prime}, x_{4}^{\prime}\right\}_{2}\right)+\mu_{2}\left(\left\{x_{1}^{\prime}, x_{3}^{\prime}, x_{4}^{\prime}\right\}_{2}, \alpha_{2}\left(x_{2}^{\prime}\right)\right) \\
& =\mu_{A_{1} \oplus A_{2}}\left(\alpha_{A_{1} \oplus A_{2}}\left(x_{1}, x_{1}^{\prime}\right),\left\{x_{2}+x_{2}^{\prime}, x_{3}+x_{3}^{\prime}, x_{4}+x_{4}^{\prime}\right\}_{A_{1} \oplus A_{2}}\right) \\
& +\mu_{A_{1} \oplus A_{2}}\left(\left\{x_{1}+x_{1}^{\prime}, x_{3}+x_{3}^{\prime}, x_{4}+x_{4}^{\prime}\right\}_{A_{1} \oplus A_{2}}, \alpha_{A_{1} \oplus A_{2}}\left(x_{2}, x_{2}^{\prime}\right)\right)
\end{aligned}
$$

This ends the proof.

Proposition 3.2. Let $\left(A_{1}, \mu_{1},\{., .,\}_{1}, \alpha_{1}\right)$ and $\left(A_{2}, \mu_{2},\{., .,\}_{2}, \alpha_{2}\right)$ be two ternary (non-commutative) Hom-Nambu-Poisson algebras. A linear map $\phi: A_{1} \rightarrow A_{2}$ is a morphism of ternary (non-commutative) HomNambu-Poisson algebras if and only if $\Gamma_{\phi} \subseteq A_{1} \oplus A_{2}$ is a Hom-NambuPoisson subalgebra of

$$
\left(A_{1} \oplus A_{2}, \mu_{A_{1} \oplus A_{2}},\{., ., .\}_{A_{1} \oplus A_{2}}, \alpha_{A_{1} \oplus A_{2}}\right)
$$

Where $\Gamma_{\phi}=\left\{(x, \phi(x)): x \in A_{1}\right\} \subset A_{1} \oplus A_{2}$.

Proof. Let $\phi:\left(A_{1}, \mu_{1},\{., .,\}_{1}, \alpha_{1}\right) \rightarrow\left(A_{2}, \mu_{2},\{., .,\}_{2}, \alpha_{2}\right)$ be a morphism of ternary

Hom-Nambu-Poisson algebras.

We have

$\left\{x_{1}+\phi\left(x_{1}\right), x_{2}+\phi\left(x_{2}\right), x_{3}+\phi\left(x_{3}\right)\right\}_{A_{1} \oplus A_{2}}=\left\{x_{1}, x_{2}, x_{3}\right\}_{1}+\left\{\phi\left(x_{1}\right), \phi\left(x_{2}\right), \phi\left(x_{3}\right)\right\}_{2}$

$=\left\{x_{1}, x_{2}, x_{3}\right\}_{1}+\phi\left\{x_{1}, x_{2}, x_{3}\right\}_{1}$

Then $\Gamma_{\phi}$ is closed under the bracket $\{. . ., .\}_{A_{1} \oplus A_{2}}$.

We have also $\left(\alpha_{1}+\alpha_{2}\right)\left(x_{1}+\phi\left(x_{1}\right)\right)=\alpha_{1}\left(x_{1}\right)+\alpha_{2}^{\circ} \phi\left(x_{1}\right)=\alpha_{1}\left(x_{1}\right)+\phi^{\circ} \alpha_{1}\left(x_{1}\right)$, which implies that $\left(\alpha_{1}+\alpha_{2}\right) \Gamma_{\phi} \subseteq \Gamma_{\phi}$.

Moreover $\Gamma_{\phi}$ is closed under the multiplication indeed

$$
\mu_{A_{1} \oplus A_{2}}\left(x_{1}+\phi\left(x_{1}\right), x_{2}+\phi\left(x_{2}\right)\right)=\mu_{1}\left(x_{1}, x_{2}\right)+\mu_{2}\left(\phi\left(x_{1}\right), \phi\left(x_{2}\right)\right)
$$$$
=\mu_{1}\left(x_{1}, x_{2}\right)+\phi^{\circ} \mu_{1}\left(x_{1}, x_{2}\right) \subseteq \Gamma_{\phi}
$$

Conversely, if the graph $\Gamma_{\phi} \subseteq A_{1} \oplus A_{2}$ is a Hom-subalgebra of

$\left(A_{1} \oplus A_{2}, \mu_{A_{1} \oplus A_{2}},\{. ., .,\}_{A_{1} \oplus A_{2}}, \alpha_{A_{1} \oplus A_{2}}\right)$,

Then we have

$\left\{\phi\left(x_{1}\right), \phi\left(x_{2}\right), \phi\left(x_{3}\right)\right\}_{2}=\phi\left\{x_{1}, x_{2}, x_{3}\right\}_{1}$,

and

$$
\begin{aligned}
& \left.\alpha_{1}+\alpha_{2}\right)(x+\phi(x))=\alpha_{1}(x)+\alpha_{2} \mathrm{o} \phi(x) \in \Gamma_{\phi} \\
& =\alpha_{1}(x)+\phi \mathrm{o} \alpha_{1}(x) .
\end{aligned}
$$

Finally

$$
\begin{aligned}
& \mu_{A_{1} \oplus A_{2}}\left(x_{1}+\phi\left(x_{1}\right), x_{2}+\phi\left(x_{2}\right)\right)=\mu_{1}\left(x_{1}, x_{2}\right)+\mu_{2}\left(\phi\left(x_{1}\right), \phi\left(x_{2}\right)\right) \\
& =\mu_{1}\left(x_{1}, x_{2}\right)+\phi \mathrm{o} \mu_{1}\left(x_{1}, x_{2}\right) \subseteq \Gamma_{\phi} .
\end{aligned}
$$

Therefore $\phi$ is a morphism of ternary (non-commutative) HomNambu-Poisson algebras.

Now, we define the tensor product of two ternary Hom-algebras. Moreover, we consider a tensor product of a ternary Hom-NambuPoisson algebra and a totally Hom-associative symmetric ternary algebra.
Let $A_{1=}(A, m, \alpha)$, where $\alpha=\left(\alpha_{i}\right)_{i=1,2}$ and $A_{2}=\left(A^{\prime}, m^{\prime}, \alpha^{\prime}\right)$ where $\alpha^{\prime}=\left(\alpha_{i}^{\prime}\right)_{i=1,2}$ be two ternary (non- commutative) Hom-algebras of a given he tensor product $A_{1} \otimes A_{1}$ is a ternary Hom-algebra defined by the triple $\left(A \otimes A^{\prime}, m \otimes m^{\prime}, \alpha \otimes \alpha^{\prime}\right)$ where $\alpha \otimes \alpha^{\prime}=\left(\alpha_{i} \otimes \alpha_{i}^{\prime}\right)_{i=1,2}$ with

$$
\begin{aligned}
& m \otimes m^{\prime}\left(x_{1} \otimes x_{1}^{\prime}, x_{2} \otimes x_{2}^{\prime}, x_{3} \otimes x_{3}^{\prime}\right)=m\left(x_{1}, x_{2}, x_{3}\right) \otimes m^{\prime}\left(x_{1}^{\prime}, x_{2}^{\prime}, x_{3}^{\prime}\right), \\
& \alpha_{i} \otimes \alpha_{i}^{\prime}\left(x_{1} \otimes x_{1^{\prime}}\right)=\alpha_{i}\left(x_{1}\right) \otimes \alpha_{i}^{\prime}\left(x_{1}^{\prime}\right), \\
& \text { Where } x_{1}, x_{2}, x_{3} \in A \text { and } x_{1}^{\prime}, x_{2}^{\prime}, x_{3}^{\prime} \in A_{2}
\end{aligned}
$$

Recall that $(A, m, \alpha)$ is a totally Hom- associative ternary algebra if

$$
\begin{aligned}
& m\left(\alpha_{1}\left(x_{1}\right), \alpha_{2}\left(x_{2}\right), m\left(x_{3}, x_{4}, x_{5}\right)\right)=m\left(\alpha_{1}\left(x_{1}\right), m\left(x_{2}, x_{3}, x_{4}\right), \alpha_{2}\left(x_{5}\right)\right) \\
& =m\left(m\left(x_{1}, x_{2}, x_{3}\right), \alpha_{1}\left(x_{4}\right), \alpha_{2}\left(x_{5}\right)\right) .
\end{aligned}
$$

for all $x_{1} \cdots, x_{5} \in A$, and the ternary multiplication $m$ is symmetric if

$$
m\left(x_{\sigma(1)}, x_{\sigma(2)}, x_{\sigma(3)}\right)=m\left(x_{1}, x_{2}, x_{3}\right) .
$$

for all $\sigma \in S_{3}, x_{1}, x_{2}, x_{3} \in A$

Lemma 3.3. Let $A_{1}=(A, m, \alpha)$ and $A_{2}=\left(A^{\prime}, m^{\prime}, \alpha^{\prime}\right)$ be two ternary Hom-algebras of given type (Hom-Nambu, totally Hom-associative). If $m$ is symmetric and $m^{\prime}$ is skew-symmetric then $m \otimes m^{\prime}$ is skew symmetric.

\section{Proof. Straight forward}

Theorem 3.4. Let $(A, \mu, \beta,\{., .,\},.(\alpha 1, \alpha 2))$ be a ternary (noncommutative) Hom- Nambu-Poisson algebra, $\left(B, \tau,\left(\alpha_{1}^{\prime}, \alpha_{2}^{\prime}\right)\right)$ be a totally Hom-associative symmetric ternary algebra, and $\left(B, \mu^{\prime}, \beta^{\prime}\right)$ be a Hom-associative algebra, then

$\left(A \otimes B, \mu \otimes \mu^{\prime}, \beta \otimes \beta^{\prime},\{., ., .\}_{A \otimes B},\left(\alpha_{1} \otimes \alpha_{1}^{\prime}, \alpha_{2} \otimes \alpha_{2}^{\prime}\right)\right)$

a (non-commutative) ternary Hom-Nambu-Poisson algebra if and only if

$$
\tau\left(\mu^{\prime}\left(b_{1}, b_{2}\right), b_{3}, b_{4}\right)=\mu^{\prime}\left(b_{1}, \tau\left(b_{2}, b_{3}, b_{4}\right)\right)=\mu^{\prime}\left(\tau\left(b_{1}, b_{3}, b_{4}\right), b_{2}\right) .
$$

Proof. Since $\mu$ and $\mu^{\prime}$ are both Hom-associative multiplication whence a tensor product $\mu \otimes \mu^{\prime}$ is Hom-associative. Also the commutativity of $\mu \otimes \mu^{\prime}$, the skew- symmetry of \{., ., .\} and the symmetry of $\tau$ simply the skew-symmetry of $\{., ., .\}_{A \otimes B}$.

Therefore, it remains to check Hom-Nambu identity and HomLeibniz identity

We have

$$
\begin{aligned}
& \text { LHS }=\left\{\alpha_{1} \otimes \alpha_{1}^{\prime}\left(a_{1} \otimes b_{1}\right), \alpha_{2} \otimes \alpha_{2}^{\prime}\left(a_{2} \otimes b_{2}\right),\left\{a_{3} \otimes b_{3}, a_{4} \otimes b_{4}, a_{5} \otimes b_{5}\right\}_{A \otimes B}\right\}_{A \otimes B} \\
& =\left\{\alpha_{1}\left(a_{1}\right) \otimes \alpha_{1}^{\prime}\left(b_{1}\right), \alpha_{2}\left(a_{2}\right) \otimes \alpha_{2}^{\prime}\left(b_{2}\right),\left\{a_{3}, a_{4}, a_{5}\right\}_{A} \otimes \tau\left(b_{3}, b_{4}, b_{5}\right)\right\}_{A \otimes B} \\
& =\{\underbrace{\left\{\alpha_{1}\left(a_{1}\right), \alpha_{2}\left(a_{2}\right),\left\{a_{3}, a_{4}, a_{5}\right\}\right\}} \otimes \underbrace{\tau\left(\alpha_{1}^{\prime}\left(b_{1}\right), \alpha_{2}^{\prime}\left(b_{2}\right), \tau\left(b_{3}, b_{4}, b_{5}\right)\right)},
\end{aligned}
$$

and

$$
\begin{aligned}
& \text { RHS }=\left\{\left\{a_{1} \otimes b_{1}, a_{2} \otimes b_{2}, a_{3} \otimes b_{3}\right\}_{A \otimes B}, \alpha_{1} \otimes \alpha_{1}^{\prime}\left(a_{4} \otimes b_{4}\right), \alpha_{2} \otimes \alpha_{2}^{\prime}\left(a_{5} \otimes b_{5}\right)\right\}_{A \otimes B} \\
& +\left\{\alpha_{1} \otimes \alpha_{1}^{\prime}\left(a_{3} \otimes b_{3}\right),\left\{a_{1} \otimes b_{1}, a_{2} \otimes b_{2}, a_{4} \otimes b_{4}\right\}_{A \otimes B}, \alpha_{2} \otimes \alpha_{2}^{\prime}\left(a_{5} \otimes b_{5}\right)\right\}_{A \otimes B} \\
& +\left\{\alpha_{1} \otimes \alpha_{1}^{\prime}\left(a_{3} \otimes b_{3}\right), \alpha_{2} \otimes \alpha_{2}^{\prime}\left(a_{4} \otimes b_{4}\right),\left\{a_{1} \otimes b_{1}, a_{2} \otimes b_{2}, a_{5} \otimes b_{5}\right\}_{A \otimes B}\right\}_{A \otimes B} \\
& =\left\{\left\{a_{1}, a_{2}, a_{3}\right\}_{A} \otimes \tau\left(b_{1}, b_{2}, b_{3}\right), \alpha_{1}\left(a_{4}\right) \otimes \alpha_{1}^{\prime}\left(b_{4}\right), \alpha_{2}\left(a_{5}\right) \otimes \alpha_{2}^{\prime}\left(b_{5}\right)\right\}_{A \otimes B} \\
& +\left\{\alpha_{1}\left(a_{3}\right) \otimes \alpha_{1}^{\prime}\left(b_{3}\right),\left\{a_{1}, a_{2}, a_{4}\right\}_{A} \otimes \tau\left(b_{1}, b_{2}, b_{4}\right), \alpha_{2}\left(a_{5}\right) \otimes \alpha_{2}^{\prime}\left(b_{5}\right)\right\}_{A \otimes B} \\
& +\left\{\alpha_{1}\left(a_{3}\right) \otimes \alpha_{1}^{\prime}\left(b_{3}\right), \alpha_{2}\left(a_{4}\right) \otimes \alpha_{2}^{\prime}\left(b_{4}\right),\left\{a_{1}, a_{2}, a_{5}\right\}_{A} \otimes \tau\left(b_{1}, b_{2}, b_{5}\right)\right\}_{A \otimes B} \\
& =\{\underbrace{\left.\left\{a_{1}, a_{2}, a_{3}\right\}, \alpha_{1}\left(a_{4}\right), \alpha_{2}\left(a_{5}\right)\right\}}_{c} \otimes \underbrace{\tau\left(\tau\left(b_{1}, b_{2}, b_{3}\right), \alpha_{1}^{\prime}\left(b_{4}\right), \alpha_{2}^{\prime}\left(b_{5}\right)\right)}_{c} \\
& +\underbrace{\left\{\alpha_{1}\left(a_{3}\right),\left\{a_{1}, a_{2}, a_{4}\right\}, \alpha_{2}\left(a_{5}\right)\right\}}_{d} \otimes \underbrace{\tau\left(\alpha_{1}^{\prime}\left(b_{3}\right), \tau\left(b_{1}, b_{2}, b_{4}\right), \alpha_{2}^{\prime}\left(b_{5}\right)\right)}_{e} \\
& +\underbrace{\left\{\alpha_{1}\left(a_{3}\right), \alpha_{2}\left(a_{4}\right),\left\{a_{1}, a_{2}, a_{5}\right\}\right\}}_{f} \otimes \underbrace{\tau(n)}_{f\left(\alpha_{1}^{\prime}\left(b_{3}\right), \alpha_{2}^{\prime}\left(b_{4}\right), \tau\left(b_{1}, b_{2}, b_{5}\right)\right)}
\end{aligned}
$$


Using ternary Nambu identity of $\{.$, ., . $\}$ we have $a=c+e+g$, and $b=d=f=h$ using the symmetry of $\tau$ and Hom-associativity of $\mu^{\prime}$, then the left hand side is equal to the right hand side from where the ternary Hom- Nambu identity of bracket $\{., ., .\}_{A \otimes B}$ is verified

For the Hom-Leibniz identity, we have

$$
\begin{aligned}
& L H S=\left\{\mu \otimes \mu^{\prime}\left(a_{1} \otimes b_{1}, a_{2} \otimes b_{2}\right), \alpha_{1} \otimes \alpha_{1}^{\prime}\left(a_{3} \otimes b_{3}\right), \alpha_{2} \otimes \alpha_{2}^{\prime}\left(a_{4} \otimes b_{4}\right)\right\}_{A \otimes B} \\
& =\left\{\mu\left(a_{1}, b_{1}\right) \otimes \mu^{\prime}\left(a_{2}, b_{2}\right), \alpha_{1}\left(a_{3}\right) \otimes \alpha_{1}^{\prime}\left(b_{3}\right), \alpha_{2}\left(a_{4}\right) \otimes \alpha_{2}^{\prime}\left(b_{4}\right)\right\}_{A \otimes B} \\
& =\underbrace{\left\{\mu\left(a_{1}, b_{1}\right), \alpha_{1}\left(a_{3}\right), \alpha_{2}\left(a_{4}\right)\right\}_{A} \otimes} \underbrace{\tau\left(\mu^{\prime}\left(a_{2}, b_{2}\right), \alpha_{1}^{\prime}\left(b_{3}\right), \alpha_{2}^{\prime}\left(b_{4}\right)\right)}
\end{aligned}
$$

And

$$
\begin{aligned}
& \text { RHS }=\mu \otimes \mu^{\prime}\left(\beta \otimes \beta^{\prime}\left(a_{1} \otimes b_{1}\right),\left\{a_{2} \otimes b_{2}, a_{3} \otimes b_{3}, a_{4} \otimes b_{4}\right\}_{A \otimes B}\right) \\
& +\mu \otimes \mu^{\prime}\left(\left\{a_{1} \otimes b_{1}, a_{3} \otimes b_{3}, a_{4} \otimes b_{4}\right\}_{A \otimes B}, \beta \otimes \beta^{\prime}\left(a_{2} \otimes b_{2}\right)\right) \\
& =\mu \otimes \mu^{\prime}\left(\beta\left(a_{1}\right) \otimes \beta^{\prime}\left(b_{1}\right),\left\{a_{2}, a_{3}, a_{4}\right\} \otimes \tau\left(b_{2}, b_{3}, b_{4}\right)\right) \\
& +\mu \otimes \mu^{\prime}\left(\left\{a_{1}, a_{3}, a_{4}\right\} \otimes \tau\left(b_{1}, b_{3}, b_{4}\right), \beta\left(a_{2}\right) \otimes \beta^{\prime}\left(b_{2}\right)\right) \\
& =\underbrace{\mu\left(\beta\left(a_{1}\right),\left\{a_{2}, a_{3}, a_{4}\right\}\right)}_{c^{\prime}} \otimes \underbrace{\mu_{1}}_{d^{\prime}\left(\beta^{\prime}\left(b_{1}\right), \tau\left(b_{2}, b_{3}, b_{4}\right)\right)} \\
& +\underbrace{\mu\left(\left\{a_{1}, a_{3}, a_{4}\right\}, \beta\left(a_{2}\right)\right)} \otimes \underbrace{\mu^{\prime}\left(\tau\left(b_{1}, b_{3}, b_{4}\right), \beta^{\prime}\left(b_{2}\right)\right.}
\end{aligned}
$$

With Hom-Leibniz identity we have $a^{\prime}=c^{\prime}+e^{\prime}$, and using condition (3.4) we have $b^{\prime}=d^{\prime}=f^{\prime}$, therefore the left hand side is equal to the right hand side and the Hom-Leibniz identity is proved. Then

$\left(A \otimes B, \mu \otimes \mu^{\prime}, \beta \otimes \beta^{\prime},\{., .,\}_{A \otimes B},\left(\alpha_{1} \otimes \alpha_{1}^{\prime}, \alpha_{2} \otimes \alpha_{2}^{\prime}\right)\right)$

is a (non-commutative) ternary Hom-Nambu-Poisson algebra.

\section{Construction of Ternary Hom-Nambu-Poisson Algebras}

In this section, we provide constructions of ternary Hom-NambuPoisson algebras using twisting principle.

Theorem 4.1. Let $(A, \mu,\{., .,\},. \alpha)$ be a (non-commutative) ternary Hom-Nambu-Poisson algebra and $\beta: A \rightarrow A$ be a weak Hom-NambuPoisson morphism, then $A_{\beta}=\left(A,\{., ., .\}_{\beta}=\beta \circ\{., .,\},. \mu \beta=\beta \circ \mu, \beta \alpha\right)$ is also a ternary (non-commutative)Hom-Nambu-Poisson algebra. Moreover, if $A$ is multiplicative and $\beta$ is an algebra morphism, then $A_{\beta}$ is a multiplicative (non-commutative) Hom-Nambu-Poisson al-gebra.

Proof. If $\mu$ is commutative, then clearly so is $\mu_{\beta}$. The rest of the proof applies whether $\mu$ is commutative or not. The skew-symmetry follows from the skew- symmetry of the bracket $\{.$, ., .\}. It remains to prove Hom-associativity condition, Hom-Nambu-identity and HomLeibniz identity. Indeed

$$
\begin{aligned}
& \mu_{\beta}\left(\mu_{\beta}(x, y), \beta \alpha(z)\right)=\mu_{\beta}(\beta(\mu(x, y), \beta \alpha(z)))=\beta^{2}(\mu(\mu(x, y), \alpha(z))) \\
& =\beta^{2}(\mu(\alpha(x), \mu(y, z)))=\mu_{\beta}\left(\beta \alpha(x), \mu_{\beta}(y, z)\right) . \\
& \text { We check the Hom-Nambu identity } \\
& \left\{\beta \alpha\left(x_{1}\right), \beta \alpha\left(x_{2}\right),\left\{x_{3}, x_{4}, x_{5}\right\}_{\beta}\right\}_{\beta}=\beta^{2}\left\{\alpha\left(x_{1}\right), \alpha\left(x_{2}\right),\left\{x_{3}, x_{4}, x_{5}\right\}\right\} \\
& =\beta^{2}\left(\left\{\left\{x_{1}, x_{2}, x_{3}\right\}, \alpha\left(x_{4}\right), \alpha\left(x_{5}\right)\right\}+\left\{\alpha\left(x_{3}\right),\left\{x_{1}, x_{2}, x_{4}\right\}, \alpha\left(x_{5}\right)\right\}\right. \\
& \left.+\left\{\alpha\left(x_{3}\right), \alpha\left(x_{4}\right),\left\{x_{1}, x_{2}, x_{5}\right\}\right\}\right) \\
& =\left\{\left\{x_{1}, x_{2}, x_{3}\right\}_{\beta}, \beta \alpha\left(x_{4}\right), \beta \alpha\left(x_{5}\right)\right\}_{\beta}+\left\{\beta \alpha\left(x_{3}\right),\left\{x_{1}, x_{2}, x_{4}\right\} \beta, \beta \alpha\left(x_{5}\right)\right\}_{\beta} \\
& +\left\{\beta \alpha(x 3), \beta \alpha\left(x_{4}\right),\left\{x_{1}, x_{2}, x_{5}\right\}_{\beta}\right\}_{\beta} .
\end{aligned}
$$

Then it remains to show Hom-Leibniz identity$$
\left\{\mu_{\beta}\left(x_{1}, x_{2}\right), \beta \alpha\left(x_{3}\right), \beta \alpha\left(x_{4}\right)\right\}_{\beta}=\beta^{2}\left(\left\{\mu\left(x_{1}, x_{2}\right), \alpha\left(x_{3}\right), \alpha\left(x_{4}\right)\right\}\right)
$$

$$
\begin{aligned}
& =\beta^{2}\left(\mu\left(\alpha\left(x_{1}\right),\left\{x_{2}, x_{3}, x_{4}\right\}\right)+\mu\left(\left\{x_{1}, x_{3}, x_{4}\right\}, \alpha\left(x_{2}\right)\right)\right) \\
& =\mu_{\beta}\left(\beta \alpha\left(x_{1}\right),\left\{x_{2}, x_{3}, x_{4}\right\}_{\beta}\right)+\mu_{\beta}\left(\left\{x_{1}, x_{3}, x_{4}\right\}_{\beta}, \beta \alpha\left(x_{2}\right)\right)
\end{aligned}
$$

Therefore $A_{\beta}=\left(A,\{., .,\}_{\beta}, \mu_{\beta}, \beta \alpha\right)$ is a ternary (non-commutative) Hom-Nambu- Poisson algebra. For the multiplicativity assertion, suppose that $A$ is multiplicative and $\beta$ is an algebra morphism. We have

$(\beta \alpha) \circ\left(\mu_{\beta}\right)=\beta \alpha o \beta o \mu=\mu_{\beta} o \alpha^{\otimes 2} \beta^{\otimes 2}=\mu_{\beta} o(\beta \alpha)^{\otimes 2}$

And

$\beta \alpha \mathrm{o}\{., ., .\}_{\beta}=\beta \alpha \mathrm{o} \beta \mathrm{o}\{., . .\}=,\{. . ., .\}_{\beta} \mathrm{o}(\beta \alpha)^{\otimes 3}$

Then $A_{\beta}$ is multiplicative.

Corollary 4.2. Let $(A, \mu,\{., .,\},. \alpha)$ be a multiplicative ternary (noncommutative) Hom-Nambu-Poisson algebra. Then

$$
A^{n}=\left(A, \mu^{(n)}=\alpha^{n} \mathrm{o} \mu,\{., .,\}^{(n)}=\alpha^{(n)} \mathrm{o}\{., ., .\}, \alpha^{n+1}\right)
$$

is a multiplicative (non-commutative) ternary Hom-Nambu-Poisson algebra for each integer $n \geq 0$.

Proof. The multiplicativity of $A$ implies that $\alpha^{n}: A \rightarrow A$ is a NambuPoisson algebra morphism. By Theorem $4.1 A_{\alpha^{n}}=A^{n}$ is a multiplicative ternary (non- commutative) Hom-Nambu-Poisson algebra.

Corollary 4.3. Let ( $A, \mu,\{., .,\}$.$) be a ternary (non-commutative)$ Nambu-Poisson algebra and $\beta: A \rightarrow A$ be a Nambu-Poisson algebra morphism. Then

$$
A_{\beta}=\left(A, \mu_{\beta}=\beta \mathrm{o} \mu,\{., .,\}_{\beta}=\beta \mathrm{o}\{., ., .\}, \beta\right)
$$

is a multiplicative (non-commutative) ternary Hom-Nambu-Poisson algebra.

Remark 4.4. Let $(A, \mu,\{., .,\},. \alpha)$ and $\left(A^{\prime}, \mu^{\prime},\{., . .,\}^{\prime}, \alpha^{\prime}\right)$ be two (non-commutative) ternary Nambu-Poisson algebras and $\beta: A \rightarrow A, \beta^{\prime}: A^{\prime} \rightarrow A^{\prime}$ be ternary Nambu- Poisson algebra endomorphisms. If $\varphi: A \rightarrow A^{\prime}$ is a ternary Nambu-Poisson algebra morphism that satisfies $\varphi \mathrm{o} \beta=\beta^{\prime} \mathrm{o} \varphi$ then

$$
\varphi:\left(A, \mu_{\beta},\{., .,\}_{\beta}, \beta \alpha\right) \rightarrow\left(A^{\prime}, \mu_{\beta^{\prime}}^{\prime},\{., .,\}_{\beta^{\prime}}^{\prime}, \beta^{\prime} \alpha^{\prime}\right)
$$

Is a (non-commutative) ternary hom-nambu poisson algebra morphism.

Indeed, we have

$$
\begin{aligned}
& \varphi \mathrm{o}\{., .,\}_{\beta}=\varphi \mathrm{o} \beta^{\circ}\{., ., .\}=\beta^{\prime} \mathrm{o} \varphi \mathrm{o}\{., ., .\}=\beta^{\prime} \mathrm{o}\{., .,\}^{\prime} \mathrm{o} \varphi^{\times 3}=\{., .,\}_{\beta^{\prime}}^{\prime \circ} \varphi^{\times 3} \\
& \varphi^{\circ} \mu_{\beta}=\varphi^{\circ} \beta^{\circ} \mu=\beta^{\prime \circ} \varphi^{\circ} \mu=\beta^{\prime \circ} \mu^{\prime \circ} \varphi^{\times 2}=\mu_{\beta^{\prime}}^{\prime} \circ \varphi^{\times 2}
\end{aligned}
$$

In the sequel, we aim to construct Hom-type version of the ternary Nambu- Poisson algebra of polynomials of three variables $(\mathbb{R}[x, y, z],,\{., .,\}$.$) , defined in Example 1.5. The Poisson bracket of$ three polynomials is defined in (1.2).

The twisted version is given by a structure of ternary Hom-NambuPoisson algebra where $\left(\mathbb{R}[x, y, z],{ }_{\alpha}=\alpha \mathrm{o},\{., .,\}_{\alpha}=\alpha 0\{., .,\},. \alpha\right)$ where $\alpha: \mathbb{R}[x, y, z] \rightarrow \mathbb{R}[x, y, z]$ is an algebra morphism satisfying for all $f, g \in \mathbb{R}[x, y, z]$

$$
\begin{aligned}
& \alpha(f \cdot g)=\alpha(f) \cdot \alpha(g) \\
& \alpha\{f, g, h\}=\{\alpha(f), \alpha(g), \alpha(h)\} .
\end{aligned}
$$


Theorem 4.5. A morphism $\quad \alpha: \mathbb{R}[x, y, z] \rightarrow \mathbb{R}[x, y, z] \quad$ which gives a structure of ternary Hom-Nambu-Poisson algebra $\left(\mathbb{R}[x, y, z],{ }_{\alpha}=\alpha^{\circ},\{, ., .,\}_{\alpha}=\alpha^{\circ}\{., .,\},. \alpha\right)$ satisfies the following equation:

$$
1-\left|\begin{array}{lll}
\frac{\partial \alpha(x)}{\partial x} & \frac{\partial \alpha(x)}{\partial y} & \frac{\partial \alpha(x)}{\partial z} \\
\frac{\partial \alpha(y)}{\partial x} & \frac{\partial \alpha(y)}{\partial y} & \frac{\partial \alpha(y)}{\partial z} \\
\frac{\partial \alpha(z)}{\partial x} & \frac{\partial \alpha(z)}{\partial y} & \frac{\partial \alpha(z)}{\partial z}
\end{array}\right|=0
$$

Proof. Let $\alpha$ be a Nambu-Poisson algebra morphism, then it satisfies for all $f, g, h \in \mathbb{R}[x, y, z]$

$$
\begin{aligned}
& \alpha(f \cdot g)=\alpha(f) \cdot \alpha(g), \\
& \alpha\{f, g, h\}=\{\alpha(f), \alpha(g), \alpha(h)\} .
\end{aligned}
$$

The first equality shows that it is sufficient to just set $\alpha$ on $x, y$ and $z$. For the second equality, we suppose by linearity that

$$
\begin{aligned}
& f(x, y, z)=x^{i} y^{j} z^{k}, \\
& g(x, y, z)=x^{l} y^{m} z^{p}, \\
& f(x, y, z)=x^{q} y^{r} z^{s} .
\end{aligned}
$$

Then we can write the second equation as follows

$$
\alpha\left|\begin{array}{lll}
\frac{\partial f}{\partial x} & \frac{\partial f}{\partial y} & \frac{\partial f}{\partial z} \\
\frac{\partial g}{\partial x} & \frac{\partial g}{\partial y} & \frac{\partial g}{\partial z} \\
\frac{\partial h}{\partial x} & \frac{\partial h}{\partial y} & \frac{\partial h}{\partial z}
\end{array}\right|=\left|\begin{array}{ccc}
\frac{\partial \alpha(f)}{\partial x} & \frac{\partial \alpha(f)}{\partial y} & \frac{\partial \alpha(f)}{\partial z} \\
\frac{\partial \alpha(g)}{\partial x} & \frac{\partial \alpha(g)}{\partial y} & \frac{\partial \alpha(g)}{\partial z} \\
\frac{\partial \alpha(h)}{\partial x} & \frac{\partial \alpha(h)}{\partial y} & \frac{\partial \alpha(h)}{\partial z}
\end{array}\right|,
$$

which can be simplified to

$$
1=\left|\begin{array}{ccc}
\frac{\partial \alpha(x)}{\partial x} & \frac{\partial \alpha(x)}{\partial y} & \frac{\partial \alpha(x)}{\partial z} \\
\frac{\partial \alpha(y)}{\partial x} & \frac{\partial \alpha(y)}{\partial y} & \frac{\partial \alpha(y)}{\partial z} \\
\frac{\partial \alpha(z)}{\partial x} & \frac{\partial \alpha(z)}{\partial y} & \frac{\partial \alpha(z)}{\partial z}
\end{array}\right| .
$$

Example 4.6. We set polynomials:

$$
\begin{aligned}
& \alpha(x)=P_{1}(x, y, z)=\sum_{0 \leq i, j, k \leq d} a_{i j k} x^{i} y^{j} z^{k}, \\
& \alpha(y)=P_{2}(x, y, z)=\sum_{0 \leq i, j, k \leq d} b_{i j k} x^{i} y^{j} z^{k}, \\
& \alpha(z)=P_{3}(x, y, z)=\sum_{0 \leq i, j, k \leq d} c_{i j k} x^{i} y^{j} z^{k},
\end{aligned}
$$

Where $P_{1}, P_{2}, P_{3} \in \mathbb{R}[x, y, z]$, and d the largest degree for each variable. We assume that $a_{0}=b_{0}=c_{0}=0$

Case of polynomials of degree one. We take

$$
\begin{aligned}
& P_{1}(x, y, z)=a_{1} x+a_{2} y+a_{3} z, \\
& P_{2}(x, y, z)=b_{1} x+b_{2} y+b_{3} z, \\
& P_{3}(x, y, z)=c_{1} x+c_{2} y+c_{3} z
\end{aligned}
$$

Equation (2.5) becomes

$$
1-\left|\begin{array}{lll}
\frac{\partial P_{1}(x, y, z)}{\partial x} & \frac{\left.\partial P_{1}(x, y, z)\right)}{\partial y} & \frac{\partial P_{1}(x, y, z)}{\partial z} \\
\frac{\partial P_{2}(x, y, z)}{\partial x} & \frac{\partial P_{2}(x, y, z)}{\partial y} & \frac{\partial P_{2}(x, y, z)}{\partial z} \\
\frac{\partial P_{3}(x, y, z)}{\partial x} & \frac{\partial P_{3}(x, y, z)}{\partial y} & \frac{\partial P_{3}(x, y, z)}{\partial z}
\end{array}\right|=0,
$$

Whence

$$
1-\left|\begin{array}{lll}
a_{1} & a_{2} & a_{3} \\
b_{1} & b_{2} & b_{3} \\
c_{1} & c_{2} & c_{3}
\end{array}\right|=0 .
$$

The polynomials $P_{1}, P_{2}$ and $P_{3}$ are of one of this form

$$
\begin{aligned}
& P_{1}(x, y, z)=x a_{1}+y a_{2}+z a_{3}, P_{2}(x, y, z)=b_{2} y-\frac{z}{a_{1} c_{2}}, P_{3}(x, y, z)=c_{2} y \text {. } \\
& P_{1}(x, y, z)=a_{1} x+a_{2} y+a_{3} z, P_{2}(x, y, z)=\frac{1+a_{1} b_{3} c_{2}}{a_{1} c_{3}} y+b_{3} z, P_{3}(x, y, z)=c_{2} y+c_{3} z \\
& P_{1}(x, y, z)=a_{1} x+a_{2} y+a_{3} z, P_{2}(x, y, z)=b_{1} x+\frac{1}{a_{2} c_{1}} z, P_{3}(x, y, z)=c_{1} x \\
& P_{1}(x, y, z)=a_{1} x+a_{2} y+a_{3} z, P_{2}(x, y, z)=\frac{-1+a_{2} b_{3} c_{1}}{a_{2} c_{3}} x+b_{3} z, P_{3}(x, y, z)=c_{1} x+c_{3} z \\
& P_{1}(x, y, z)=\frac{a_{2} b_{1} c_{3}+b_{2}}{c_{3} x}+a_{2} y+a_{3} z, P_{2}(x, y, z)=b_{1} x+b_{2} y+b_{3} z, P_{3}(x, y, z)=c_{3} z \\
& P_{1}(x, y, z)=\frac{1}{b_{2} c_{3}} x+a_{2} y+a_{3} z, P_{2}(x, y, z)=b_{2} y+b_{3} z, P_{3}(x, y, z)=c_{3} z \\
& P_{1}(x, y, z)=a_{1} x+\frac{1}{b_{1} c_{3}} y+a_{3} z, P_{2}(x, y, z)=b_{1} x+b_{3} z, P_{3}(x, y, z)=c_{3} z \\
& P_{1}(x, y, z)=a_{1} x+a_{2} y+\frac{1}{b_{1} c_{2}} z, P_{2}(x, y, z)=b_{1} x, P_{3}(x, y, z)=c_{1} x+c_{2} y \\
& P_{1}(x, y, z)=a_{1} x+\frac{-1}{b_{1} c_{3}+a_{3} c_{2} c_{3}} y+a_{3} z, P_{2}(x, y, z)=b_{1} x, P_{3}(x, y, z)=c_{1} x+c_{2} y+c_{3} z . \\
& P_{1}(x, y, z)=\frac{a_{2} b_{1}}{b_{2}}+\frac{1}{b_{2} c_{3}-b_{3} c_{2}} x+a_{2} y+a_{3} z, P_{2}(x, y, z)=b_{1} x+b_{2} y+b_{3} z, P_{3}(x, y, z)=\frac{b_{1} c_{2}}{b_{2}} x+c_{2} y+c_{3} z \\
& P_{1}(x, y, z)=\frac{-c_{3}+a_{2} c_{1} c_{2}}{b_{3} c_{2}^{2}} x+a_{2} y+a_{3} z, P_{2}(x, y, z)=b_{3} z, P_{3}(x, y, z)=c_{1} x+c_{2} y+c_{3} z \\
& P_{1}(x, y, z)=a_{1} x+a_{2} y+\frac{1}{b_{1} c_{2}-b_{2} c_{1}} z, P_{2}(x, y, z)=b_{1} x+b_{2} y, P_{3}(x, y, z)=c_{1} x+c_{2} y \\
& P_{1}(x, y, z)=\frac{1+a_{2} b_{1} c_{3}-a_{3} b_{1} c_{2}-a_{2} b_{3} c_{1}+a_{3} b_{2} c_{1}}{b_{2} c_{3}-b_{3} c_{2}} x+a_{2} y+a_{3} z, P_{2}(x, y, z)=b_{1} x+b_{2} y+ \\
& P_{1}(x, y, z)=a_{1} x+\frac{b_{2}}{b_{3}}\left(a_{3}-\frac{1}{b_{1} c_{2}-b_{2} c_{1}}\right) y+a_{3} z, P_{2}(x, y, z)=b_{1} x+b_{2} y+b_{3} z, P_{3}(x, y, z)
\end{aligned}
$$

Particular case of polynomials of degree two. We take one of the polynomials of degree two

$$
\begin{aligned}
& P_{1}(x, y, z)=a_{1} x+a_{2} y+a_{3} z \\
& P_{2}(x, y, z)=b_{1} x+b_{2} y+b_{3} z \\
& P_{3}(x, y, z)=c_{1} x+c_{2} y+c_{3} z+c_{4} x^{2}
\end{aligned}
$$

The polynomials $P_{1}, P_{2}$ and $P_{3}$ are of one of this form

$$
P_{1}(x, y, z)=\frac{a_{2} b_{1}}{b_{2}}+\frac{1}{b_{2} c_{3}-b_{3} c_{2}} x+a_{2} y+\frac{a_{2} b_{3}}{b_{2}} z P_{2}(x, y, z)=b_{1} x+b_{2} y+b_{3} z
$$




$$
\begin{aligned}
& P_{1}(x, y, z)=a_{2} x+\frac{a_{3} b_{2}}{b_{3}} y+a_{3} z, P_{2}(x, y, z)=b_{2} y+b_{3} z, P_{3}(x, y, z)=c_{4} x^{2}+c_{1} x+c_{2} y+\frac{\frac{1}{a_{1}}+b_{3} c_{2}}{b_{2}} z \\
& P_{1}(x, y, z)=a_{2} x+a_{2} y+a_{3} z, P_{2}(x, y, z)=b_{2} y, P_{3}(x, y, z)=c_{4} x^{2}+c_{1} x+c_{2} y+\frac{1}{a_{1} b_{2}} z . \\
& P_{1}(x, y, z)=\left(\frac{a_{2} b_{1}}{b_{3}}-\frac{1}{c_{2} b_{3}}\right) x+a_{3} z, P_{2}(x, y, z)=b_{1} x+b_{3} z, P_{3}(x, y, z)=c_{4} x^{2}+c_{1} x+c_{2} y+c_{3} z \\
& P_{1}(x, y, z)=-\frac{1}{b_{3} c_{2}} x+a_{3} z, P_{2}(x, y, z)=b_{3} z, P_{3}(x, y, z)=c_{4} x^{2}+c_{1} x+c_{2} y+c_{3} z . \\
& P_{1}(x, y, z)=a_{1} x-\frac{1}{b_{1} c_{3}} y+a_{3} z, P_{2}(x, y, z)=b_{1} x, P_{3}(x, y, z)=c_{4} x^{2}+c_{1} x+c_{3} z \\
& P_{1}(x, y, z)=a_{1} x+\frac{-1}{b_{1} c_{3}}+\frac{a_{3} c_{2}}{c_{3}} y+a_{3} z, P_{2}(x, y, z)=b_{1} x, P_{3}(x, y, z)=c_{4} x^{2}+c_{1} x+c_{2} y+c_{3} z \\
& P_{1}(x, y, z)=a_{1} x+a_{2} y+\frac{1}{b_{1} c_{2}} z, P_{2}(x, y, z)=b_{1} x, P_{3}(x, y, z)=c_{4} x^{2}+c_{1} x+c_{2} y \\
& P_{1}(x, y, z)=a_{1} x+a_{2} y+a_{3} z, P_{2}(x, y, z)=\frac{\left(1+a_{2} b_{3} c_{1}\right)}{a_{2} c_{3}} x+b_{3} z, P_{3}(x, y, z)=c_{1} x+c_{3} z
\end{aligned}
$$

\section{Classification}

In this section, we provide the classification of 3-dimensional ternary non-commutative Nambu-Poisson algebras. By straightforward calculations and using a computer algebra system we obtain the following result.

Theorem 5.1. Every 3-dimensional ternary Nambu-Lie algebra is isomorphic to the ternary algebra defined with respect to basis $\left\{e_{1}, e_{2}, e_{3}\right\}$, by the skew-symmetric bracket defined as

$$
\left\{e_{1}, e_{2}, e_{3}\right\}=e_{1}
$$

Moreover it defines a 3-dimensional ternary non-commutative Nambu-Poisson algebra (A, \{., ., .\}, $\mu$ ) if and only if $\mu$ is one of the following non-commutative associative algebra defined as

$$
\begin{aligned}
& \mu_{1}\left(e_{2}, e_{1}\right)=a e_{1} \mu_{1}\left(e_{2}, e_{2}\right)=a e_{2} \mu_{1}\left(e_{2}, e_{3}\right)=a e_{3} \\
& \mu_{1}\left(e_{3}, e_{1}\right)=b e_{1} \mu_{1}\left(e_{3}, e_{2}\right)=b e_{2} \mu_{1}\left(e_{3}, e_{3}\right)=b e_{3},
\end{aligned}
$$

where $a, b$ are parameters.

The opposite algebra of (1)

The multiplication which are not mentioned are equal to zero.

The first statement of the Theorem is due to Filippov [4,13]. The two families are naturally not isomorphic.

Remark 5.2. The 3-dimensional ternary Nambu-Lie algebra is endowed with a commutative Nambu-Poisson algebra structure only when the multiplication is trivial.

Using the twisting principle described in Theorem 4.1, we obtain the following 3-dimensional non-commutative ternary Hom-NambuPoisson algebras.

Proposition 5.3. Any 3-dimensional ternary non-commutative Hom-Nambu-Poisson algebra $\left(A,\{., .,\}_{\alpha}, \mu_{\alpha}, \alpha\right)$ obtained by a twisting defined with respect to the basis $\left\{e_{1}, e_{2}, e_{3}\right\}=e_{1}$ by the ternary bracket $\left\{e_{1}, e_{2}, e_{3}\right\}_{\alpha}=c e_{1}$, where $c$ is a parameter, is one of the following binary Hom-associative algebras defined by $\mu_{\alpha_{i}}$ and a corresponding structure map

$$
\begin{array}{ll}
\mu_{\alpha_{1}}\left(e_{2}, e_{1}\right)=a c e_{1}, & \mu_{\alpha_{1}}\left(e_{3}, e_{1}\right)=b c e_{1}, \\
\mu_{\alpha_{1}}\left(e_{2}, e_{2}\right)=a\left(d e_{1}+e_{2}\right), & \mu_{\alpha_{1}}\left(e_{3}, e_{2}\right)=b\left(d e_{1}+e_{2}\right), \\
\mu_{\alpha_{1}}\left(e_{2}, e_{3}\right)=a\left(h e_{1}+g e_{2}+e_{3}\right), & \mu_{\alpha_{1}}\left(e_{3}, e_{3}\right)=b\left(h e_{1}+g e_{2}+e_{3}\right),
\end{array}
$$

With

$$
\begin{array}{ll}
\alpha_{1}\left(e_{1}\right)=c e_{1}, \alpha_{1}\left(e_{2}\right)=d e_{1}+e_{2}, \alpha_{1}\left(e_{3}\right)=h e_{1}+g e_{2}+e_{3} . & \\
\mu_{\alpha_{2}}\left(e_{1}, e_{2}\right)=a c e_{1}, & \mu_{\alpha_{2}}\left(e_{3}, e_{1}\right)=b c e_{1}, \\
\mu_{\alpha_{2}}\left(e_{2}, e_{2}\right)=a\left(d e_{1}+e_{2}+l e_{3}\right), & \mu_{\alpha_{2}}\left(e_{3}, e_{2}\right)=b\left(d e_{1}+e_{2}+l e_{3}\right), \\
\mu_{\alpha_{2}}\left(e_{2}, e_{3}\right)=a\left(h e_{1}+e_{3}\right), & \mu_{\alpha_{2}}\left(e_{3}, e_{3}\right)=b\left(h e_{1}+e_{3}\right),
\end{array}
$$

With

$$
\begin{array}{ll}
\alpha_{2}\left(e_{1}\right)=c e_{1}, \alpha_{2}\left(e_{2}\right)=d e_{1}+e_{2}+l e_{3}, \alpha_{2}\left(e_{3}\right)=h e_{1}+e_{3} e_{3} \\
\begin{array}{ll}
\mu_{\alpha_{3}}\left(e_{2}, e_{1}\right)=a c e_{1}, & \mu_{\alpha_{3}}\left(e_{3}, e_{1}\right)=b c e_{1}, \\
\mu_{\alpha_{3}}\left(e_{2}, e_{2}\right)=a\left(d e_{1}+f e_{2}+\frac{a}{b}(1-f) e_{3}\right), & \mu_{\alpha_{3}}\left(e_{3}, e_{2}\right)=b d e_{1}+b f e_{2}+a(1-f) e_{3}, \\
\left.\mu_{\alpha_{3}}\left(e_{2}, e_{3}\right)=a h e_{1}+b(f-1) e_{2}+\frac{a(b-g a)}{b} e_{3}\right), & \mu_{\alpha_{3}}\left(e_{3}, e_{3}\right)=b h e_{1}+\frac{b^{2}(f-1)}{a} e_{2}+(b-g a) e_{3},
\end{array}
\end{array}
$$

With

$\alpha_{3}\left(e_{1}\right)=c e_{1}, \alpha_{3}\left(e_{2}\right)=d e_{1}+f e_{2}+\frac{a}{b}(1-f) e_{3}, \alpha_{3}\left(e_{3}\right)=h e_{1}+\frac{b}{a}(f-1) e_{2}+\frac{(b-g a)}{b} e_{3}$

$$
\begin{array}{ll}
\mu_{\alpha_{4}}\left(e_{1}, e_{2}\right)=a c e_{1}, & \mu_{\alpha_{4}}\left(e_{2}, e_{3}\right)=b\left(d e_{1}+e_{2}\right), \\
\mu_{\alpha_{4}}\left(e_{1}, e_{3}\right)=b c e_{1}, & \mu_{\alpha_{4}}\left(e_{3}, e_{2}\right)=a\left(h e_{1}+g e_{2}+e_{3}\right), \\
\mu_{\alpha_{4}}\left(e_{2}, e_{2}\right)=a\left(d e_{1}+e_{2}\right), & \mu_{\alpha_{4}}\left(e_{3}, e_{3}\right)=b\left(h e_{1}+g e_{2}+e_{3}\right),
\end{array}
$$

With

$$
\begin{array}{ll}
\alpha_{4}\left(e_{1}\right)=c e_{1}, \alpha_{4}\left(e_{2}\right)=d e_{1}+e_{2}, & \alpha_{4}\left(e_{3}\right)=h e_{1}+g e_{2}+e_{3} . \\
\mu_{\alpha_{5}}\left(e_{1}, e_{2}\right)=a c e_{1}, & \mu_{\alpha_{5}}\left(e_{2}, e_{3}\right)=b\left(d e_{1}+e_{2}+l e_{3}\right), \\
\mu_{\alpha_{5}}\left(e_{1}, e_{3}\right)=b c e_{1}, & \mu_{\alpha_{5}}\left(e_{3}, e_{2}\right)=a\left(h e_{1}+e_{3}\right), \\
\mu_{\alpha_{5}}\left(e_{2}, e_{2}\right)=a\left(d e_{1}+e_{2}+l e_{3}\right), & \mu_{\alpha_{5}}\left(e_{3}, e_{3}\right)=b\left(h e_{1}+e_{3}\right)
\end{array}
$$

With

$\alpha_{5}\left(e_{1}\right)=c e_{1}, \alpha_{5}\left(e_{2}\right)=d e_{1}+e_{2}+l e_{3}, \alpha_{5}\left(e_{3}\right)=h e_{1}+e_{3}$

$$
\begin{array}{lrl}
\mu_{a_{0}}\left(e_{1}, e_{2}\right)=a c e_{1}, & \left.\mu_{a_{s}}\left(e_{2}, e_{3}\right)=b d e_{1}+b f e_{2}+a(1-f) e_{3}\right), \\
\mu_{a_{0}}\left(e_{1}, e_{3}\right)=b c e_{1}, & \mu_{a_{s}}\left(e_{3}, e_{2}\right)=a h e_{1}-b(f-1) e_{2}+\frac{a(b-a g)}{b} e_{3}, \\
\mu_{a_{0}}\left(e_{2}, e_{2}\right)=a\left(d e_{1}+f e_{2}+\frac{a}{b}(1-f) e_{3}\right), & \mu_{\alpha_{b}}\left(e_{3}, e_{3}\right)=b h e_{1}-\frac{b^{2}(f-1)}{a} e_{2}+(b-a g) e_{3}
\end{array}
$$

With

$\alpha_{6}\left(e_{1}\right)=c e_{1}, \alpha_{6}\left(e_{2}\right)=d e_{1}+f e_{2}+\frac{a}{b}(1-f) e_{3}, \alpha_{6}\left(e_{3}\right)=h e_{1}+\frac{-b}{a}(f-1) e_{2}+\frac{b-a g}{b} e_{3} z 8$

$\mu_{\alpha_{7}}\left(e_{1}, e_{3}\right)=a c e_{1}$,

$\mu_{\alpha_{7}}\left(e_{2}, e_{3}\right)=a\left(d e_{1}+f e_{2}+l e_{3}\right)$,

$\mu_{\alpha_{7}}\left(e_{3}, e_{3}\right)=a\left(h e_{1}+g e_{2}+\frac{1+g+l}{f} e_{3}\right)$

With

$$
\begin{aligned}
& \alpha_{7}\left(e_{1}\right)=c e_{1}, \alpha_{7}\left(e_{2}\right)=d e_{1}+f e_{2}+l e_{3}, \alpha_{7}\left(e_{3}\right)=h e_{1}+g e_{2}+\frac{1+g+l}{f} e_{3} \\
& \mu_{\alpha_{8}}\left(e_{1}, e_{3}\right)=a c e_{1}, \\
& \mu_{\alpha_{8}}\left(e_{2}, e_{3}\right)=a\left(d e_{1}+e_{2}\right), \\
& \mu_{\alpha_{8}}\left(e_{3}, e_{3}\right)=a\left(h e_{1}+g e_{2}+e_{3}\right)
\end{aligned}
$$

With

$\alpha_{8}\left(e_{1}\right)=c e_{1}, \alpha_{8}\left(e_{2}\right)=d e_{1}+e_{2}, \alpha_{8}\left(e_{3}\right)=h e_{1}+g e_{2}+e_{3}$.

$\mu_{\alpha_{9}}\left(e_{1}, e_{3}\right)=a c e_{1}$,

$\mu_{\alpha_{9}}\left(e_{2}, e_{3}\right)=a\left(d e_{1}-\frac{1}{g} e_{3}\right)$,

$\mu_{\alpha_{9}}\left(e_{3}, e_{3}\right)=a\left(h e_{1}+g e_{2}+r e_{3}\right)$

With

$\alpha_{9}\left(e_{1}\right)=c e_{1}, \alpha_{9}\left(e_{2}\right)=d e_{1}-\frac{1}{g} e_{3}, \alpha_{9}\left(e_{3}\right)=h e_{1}+g e_{2}+r e_{3}$ 
Citation: Makhlouf A, Amri A (2015) Non-Commutative Ternary Nambu-Poisson Algebras and Ternary Hom-Nambu-Poisson Algebras. J Generalized Lie Theory Appl 9: 221. doi:10.4172/1736-4337.1000221

Page 8 of 8

\section{References}

1. Nambu Y (1973) Generalized hamiltonian mechanics. Phys Rev D 7: 24052412.

2. Dito G, Flato M, Sternheimer D, Takhtajan L (1997) Deformation quantization and Nambu mechanics. Commun Math Phys 183: 1-22.

3. Takhtajan L (1994) On foundation of the generalized Nambu mechanics. Comm Math Phys 160: 295-315.

4. Filippov VT (1985) n-Lie algebras. Siberian Math J 26: 879-891.

5. Ataguema H, Makhlouf A, Silvestrov S (2009) Generalization of n-ary Nambu algebras and beyond. J Math Phys.

6. Hartwig JT, Silvestrov DLS (2003) Deformations of lie algebras using $\sigma$-derivations. Journal of Algebra 295: 314-361.
7. Makhlouf A Silvestrov SD (2008) Hom-algebra structures. Journal of Generalized Lie Theory and Applications 2: 51-64.

8. Yau D (2010) Non-commutative Hom-poisson algebras.

9. Ammar F, Makhlouf A Silvestrov S (2010) Ternary q-virasoro-witt Hom-NambuLie algebras. Journal of Physics A: Mathenmetical and Theoritical.

10. Ammar A, Mabrouk S, Makhlouf A (2011) Representations and cohomology of n-ary multiplicative Hom-Nambu-Lie algebras. J Geom Physics 61: 1898-1913.

11. Arnlind J, Makhlouf A, Silvestrov S (2010) Ternary Hom-Nambu-Lie algebras induced by Hom-Lie algebras, J Math Phys.

12. Yau D (2012) On n-ary Hom-Nambu and Hom-Nambu-Lie algebras. J Geom Phys 62: 506-522.

13. Yau D (2010) A Hom-associative analogue of $n$-ary Hom-Nambu algebras. 